\title{
DAMAGE DETECTION FOR HISTORICAL ARCHITECTURES BASED ON TLS INTENSITY DATA
}

\author{
Quan $\mathrm{Li}^{1,2, *}$, Xiaojun Cheng ${ }^{1}$ \\ ${ }^{1}$ College of Surveying and Geo-informatics, Tongji University, Shanghai 200092, China - (329yvonnelee, cxj)@tongji.edu.cn \\ ${ }^{2}$ Beijing Key Laboratory of Urban Spatial Information Engineering, Beijing 100038, China
}

Commission III, WG III/5

KEY WORDS: Terrestrial Laser Scanning, Intensity, Correction, Damage detection, Classification

\begin{abstract}
:
TLS (Terrestrial Laser Scanner) has long been preferred in the cultural heritage field for 3D documentation of historical sites thanks to its ability to acquire the geometric information without any physical contact. Besides the geometric information, most TLS systems also record the intensity information, which is considered as an important measurement of the spectral property of the scanned surface. Recent studies have shown the potential of using intensity for damage detection. However, the original intensity is affected by scanning geometry such as range and incidence angle and other factors, thus making the results less accurate. Therefore, in this paper, we present a method to detect certain damage areas using the corrected intensity data. Firstly, two data-driven models have been developed to correct the range and incidence angle effect. Then the corrected intensity is used to generate $2 \mathrm{D}$ intensity images for classification. After the damage areas being detected, they are re-projected to the 3D point cloud for better visual representation and further investigation. The experiment results indicate the feasibility and validity of the corrected intensity for damage detection.
\end{abstract}

\section{INTRODUCTION}

Historical architectures as a nation's cultural heritage, representing a country's cultural legacy, its conservation and management are no doubt of great significance. In the last few decades, numbers of researchers have contributed to the conservation of cultural heritage and most of them focused their attention on 3D documentation to achieve highly detailed 3D models. The development and growth of the 3D survey techniques, from photogrammetry to laser scanning, from ground-based to UAVs (Unmanned Aerial Vehicles) have also contributed greatly the 3D documentation of cultural heritage. TLS with its completeness, accuracy and fastness characteristics is rapidly becoming one of the most commonly used techniques in the heritage conservation field. It can not only protect cultural heritage but also facilitate the understanding of their present state, construction and rehabilitation, and exhibition and promotion. Most previous research efforts have been exerted to investigate the geometrical information obtained by TLS in order to generate more accurate and realistic 3D models of the historical sites. In addition to geometric information, most TLS systems also record intensity data which is the information represents the peak amplitudes recorded in the laser backscattering beam return from the scanned surface. The intensity data can be utilized to interpret the scattering properties of the scanned surface and has shown its potential in a variety of fields like forestry inventory (Zhu et al., 2015), aeolian environment monitoring (Nield et al., 2014) and geology (Hartzell et al., 2014; Carrea et al., 2016). In addition, some researchers also investigated the application of intensity in monitoring the health state of historical architectures. Armesto et al. (2010) first investigated the potential of intensity data for the detection and classification of damages in masonry structures of a historical building. Riveiro et al. (2016) applied the intensity images to conduct segmentation of masonry blocks, which may be helpful for the future stability analysis of the historic structure. Most of these studies utilized the original intensity data acquired. However, many studies indicate that the intensity data is not merely influenced by target surface characteristics. Other factors like scanning geometry, scanner mechanism and surrounding environment may also affect the intensity (Kashani et al., 2015). Therefore, these results achieved using original intensity may be less accurate and trustworthy. In order to get a more credible result, we proposed a method that utilized the corrected intensity data for the damage detection of historical architectures in this paper. We investigated the intensity data correction method first. Then it was applied to the study areas, some inner walls of an ancient Chinese pagoda. After intensity correction, the 3D point cloud was projected to $2 \mathrm{D}$ intensity images for image classification to detect certain damage areas. Finally, the damage areas detected were re-projected to the $3 \mathrm{D}$ point cloud for better visual representation and further investigation.

The remainder of this paper is organized as follows. We first present a review of intensity data correction and damage detection for historical architectures in Section 2. The proposed methods are described in Section 3. Section 4 describes the experiments conducted. Finally, conclusions follow in Section 5.

\section{RELATED WORK}

\subsection{Intensity Data Correction}

Researches focused on the correction of intensity data date back to a decade ago and numerous procedures and methods have

\footnotetext{
* Corresponding author
} 
been proposed. These methods are mainly divided into two categories: model-driven method and data-driven method. (Höfle and Pfeifer, 2007). Model-driven method is based on the physical theory of the LiDAR equation, which relates the received laser power with the transmitted power, system efficiency and geometry, etc. This kind of method can achieve results with physical meanings but it does not compensate for systematic errors and other noises during scanning. Different from model-driven method, in data-driven method the actual physical transmission of laser is dismissed. This method first constructs a suitable relationship between the transmitted laser power with the received power and several effective factors. Intensity data from homogenous region is then extracted and examined. Through some form of best-fit equation, the model parameters can be computed (Ding et al., 2013). Data-driven method is easy to conduct but it has certain limitations in intercomparison of instruments. Both model and data-driven methods have been successfully developed for ALS intensity data correction (Habib et al., 2011; Jutzi and Gross, 2014). Compared with ALS data, only a few recent studies have focused on the TLS intensity correction. And its correction also faces more unique challenges than ALS data ( $\mathrm{Li}$ et al., 2016). Many studies have found that the intensity data does not follow the LiDAR equation in the near range and different TLS systems may result in different intensity-range relations (Kaasalainen et al., 2011; Fang et al., 2014; Tan and Cheng, 2016). Therefore, most TLS intensity correction studies investigated data-driven method instead of the model-driven one (Tan et al., 2016; Blaskow and Schneider, 2014).

\subsection{Damage Detection for Historical Architectures}

Historical architectures often suffer certain damages due to natural factors, human vandalism and lack of proper maintenance. There are different kinds of damages which affect the historical architectures, including moisture, weathering, salt blooming and biological changes like moss and lichen (Armesto et al., 2010). These kinds of material damage need to be detected and monitored carefully. Traditional damage detection of historical architectures often needs manual analysis which is often performed by an expert through naked eyes. This kind of method is labour-intense and time-consuming. Moreover, some heritages are not allowed to directly touch or intervene in order to avoid erosion caused by the detection devices or human skins (Moses et al., 2014). 2D image-based techniques are also commonly used for detection of damages. Close-range photogrammetry has been widely used in 3D documentation of cultural heritage thanks to its flexibility in data acquisition and easy operability. The acquired RGB images can be useful for skilled experts to locate and quantify the damaged areas. The 2D images acquired by the infrared camera are also used. These images may be helpful for the detection of certain damages which are not visible in RGB images (Kedzierski et al., 2017). However, the images often have small size which can only be used in limited areas. Moreover, the infrared images are prone to errors caused by temperature and other environment effects. TLS is often used for 3D documentation purpose in heritage conservation filed, where the geometric information is often used. In recent years, the use of intensity data is also increasing. In the research of Armesto et al. (2010), they combined the intensity value of three different laser scanners with three kinds of unsupervised classification methods to detect and quantify the damages that affect building materials of the historical buildings. Garcíatalegónet al. (2015) investigated the usage of intensity data provided by different terrestrial laser scanners for detection and assessing of pathologies in facades. Lerones et al.
(2016) investigated the moisture detection of historical building using TLS intensity data. All these studies indicate that the intensity data can be a useful tool to assess the state of conservation of materials of the historical buildings. However, in most of their studies the intensity data used was not corrected thus making their results less accurate and trustworthy.

\section{PROPOSED METHODS}

\subsection{Intensity Data Correction}

The scanning geometry including the range between the scanner and target and incidence angle is the major factor that influence the original intensity data (Kashani et al., 2015). Therefore, in most previous work, correcting for scanning geometry was the main focus. As it was indicated in the related work section, the model-driven model is not appropriate for TLS intensity. Therefore, the data-driven model is chosen for intensity correction in this paper. Intensity correction means to convert the original intensity data $I$ into a corrected value that is proportional or equal to the target reflectance (Höfle and Pfeifer, 2007). According to the definition, the corrected intensity data $I_{c}$ can be derived as follows:

$$
I_{c}=I \frac{f_{1}\left(R_{s}\right) f_{2}\left(\theta_{s}\right)}{f_{1}(R) f_{\lambda}(\theta)}
$$

Where $f_{1}(*)=$ approximated intensity as a function of range

$f_{2}(*)=$ approximated intensity as a function of incidence angle

$R$ = range between the scanner and target

$R_{s}=$ the reference range

$\theta=$ incidence angle

$\theta_{s}=$ the reference incidence angle

In order to correct the intensity data, the specific forms of $f_{1}(\cdot)$ and $f_{2}(\cdot)$ need to be estimated. Since the distance effect and the incidence effect are unrelated, so these two functions can be estimated separately.

\subsection{D Intensity Image Generation}

The 3D point cloud can be transformed to $2 \mathrm{D}$ intensity images based on central projection. As illustrated in Figure 1, $O$ is the scanning centre, $P(x, y, z)$ is one of the point in the point cloud. $P^{\prime}$ is a point when $P$ is projected to plane $X O Y, r$ is the range between the scanning centre and $P$ and $\theta, \varphi$ are the angles between $O P^{\prime}$ and $O P, O X$ respectively.

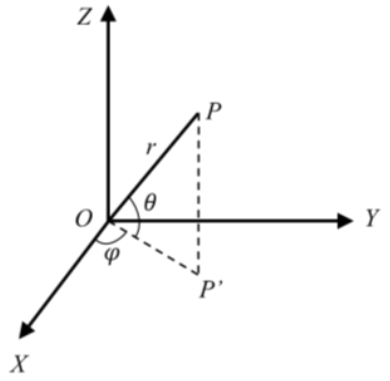

Figure 1. Point projection

The 3D point cloud can be generated to an intensity image according to the following steps: 
1. Calculate $r, \theta$ and $\varphi$ of each point in the point cloud using the following formula:

$$
\left\{\begin{array}{l}
\tan \theta=\frac{y}{x} \\
r=\sqrt{x^{2}+y^{2}+z^{2}} \\
\sin \varphi=\frac{z}{r}
\end{array}\right.
$$

2. Based on the calculation results of formula (2), the maximum and minimum value of $\theta$ and $\varphi$ can be derived. Set a sampling interval $\Delta$ and the total number of image rows $M$ and columns $N$ can be calculated:

$$
\left\{\begin{array}{l}
M=\operatorname{int}\left(\frac{\theta_{\max }-\theta_{\min }}{\Delta}\right)+1 \\
N=\operatorname{int}\left(\frac{\varphi_{\max }-\varphi_{\min }}{\Delta}\right)+1
\end{array}\right.
$$

3. Calculate the row number nrow and the column number ncol of each point according to formula (4) and these two numbers are stored for the 3D re-projection purpose:

$$
\left\{\begin{array}{l}
\text { nrow }=M-\frac{i \operatorname{int}\left(\varphi-\varphi_{\min }\right)}{\Delta} \\
\text { ncol }=N-\frac{\operatorname{int}\left(\theta-\theta_{\min }\right)}{\Delta}
\end{array}\right.
$$

4. Calculate the pixel value using the following formula (5). If there exist more than one point in a pixel, the average intensity of these points will be used in the calculation:

$$
\frac{I-I_{\min }}{I_{\max }-I_{\min }}=\frac{G-G_{\min }}{G_{\max }-G_{\min }}
$$

Where $\left[I_{\min }, I_{\max }\right]_{=}$original intensity interval $\left[G_{\min }, G_{\max }\right]=$ intensity after linear extension

\subsection{Image Classification}

The k-means algorithm is used for image classification in this paper. It is one of the simplest unsupervised learning algorithms that can classify a given data set through a certain number of clusters (assume $k$ clusters) fixed a priori. The algorithm is mainly composed of the following steps:

1. Define $k$ centroids, one for each cluster. In order to derive a better result, these centroids should be placed as much far as possible.

2. Take each point belonging to a given data set and associate it to the nearest centroid

3. When all points have been assigned a cluster, recalculate the position of the $k$ centroids

4. Repeat step 2 and step 3 until the location of the $k$ centroids no longer changes. This produces a separation of different clusters into groups from which the metric to be minimized in the following formula can be calculated:

$$
\mathrm{J}=\sum_{j=1}^{k} \sum_{i=1}^{n}\left\|x_{i}^{(j)}-c_{j}\right\|^{2}
$$

Where

$$
\begin{aligned}
& x_{i}^{(j)}=\text { a data point } \\
& c_{j}=\text { the cluster centre } \\
& \left\|x_{i}^{(j)}-c_{j}\right\|^{2}=\text { a chosen distance measure between } \\
& \quad x_{i}^{(j)} \text { and } c_{j} \\
& n=\text { the number of the data points }
\end{aligned}
$$

\section{EXPERIMENTS}

\subsection{Range and Incidence Angle Effects Experiments}

To estimate the parameters of the respect range-intensity and incidence angle-intensity polynomial functions, two sets of experiments have been conducted. The experiments were conducted under laboratory conditions with the Faro Focus ${ }^{3 \mathrm{D}}$ 120 terrestrial laser scanner. The main system parameters of the scanner are listed in Table 1 . The equipment utilized in the experiments are illustrated in Figure 2. A planar target printed on an A3 paper was used as the scanning target, which can be considered as a Lambertian target. The paper was fixed on the centre of a blackboard, on the side of which a goniometer was placed to enable the blackboard to rotate in a certain angle.

\begin{tabular}{|l|c|}
\hline System parameters & Settings \\
\hline Emitted power & $20 \mathrm{~mW}$ \\
Wavelength & $905 \mathrm{~nm}$ \\
Beam divergence & $0.009^{\circ}$ \\
Maximum range & $120 \mathrm{~m}$ \\
Field of view & $360^{\circ} \times 305^{\circ}$ \\
\hline
\end{tabular}

Table 1. Main system parameters of Faro Focus ${ }^{3 \mathrm{D}} 120$

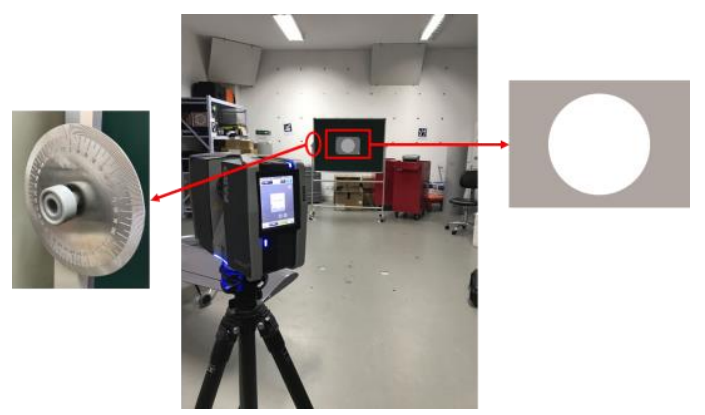

Figure 2. Equipment utilized in the experiments

4.1.1 Range Effect Correction: The experiment was implemented at a fixed incidence angle of $0^{\circ}$ from the scanner and target was set to move in steps of $0.25 \mathrm{~m}$ from $1 \mathrm{~m}$ to $10 \mathrm{~m}$, $0.5 \mathrm{~m}$ from $10 \mathrm{~m}$ to $15 \mathrm{~m}$ and $1 \mathrm{~m}$ from $15 \mathrm{~m}$ to $30 \mathrm{~m}$. For the range effect correction experiment, the scan resolution is set to $1 / 4$, and the scan quality is set to 4 with a default field of view of $360^{\circ} \times 305^{\circ}$. The intensity data of the target are extracted by Faro Scene manually. The result of the range effect experiment is illustrated in Figure 3. The intensity data first decreases as the range increases from $1 \mathrm{~m}$ to $2.5 \mathrm{~m}$, then it increases from $2.5 \mathrm{~m}$ to $5.5 \mathrm{~m}$, followed by a steep decrease from $5.5 \mathrm{~m}$ to $14 \mathrm{~m}$. Finally, the intensity begins to level out for ranges over $14 \mathrm{~m}$. Therefore, the estimation function can be written as follows:

$$
f_{1}(R)= \begin{cases}a_{1} R^{3}+b_{1} R^{2}+c_{1} R+d_{1}, & R \leq 2.5 \\ a_{2} R^{3}+b_{2} R^{2}+c_{2} R+d_{2}, & R \leq 5.5 \\ a_{3} R^{3}+b_{3} R^{2}+c_{3} R+d_{3}, & R \leq 14 \\ a_{4} R^{3}+b_{4} R^{2}+c_{4} R+d_{4}, & R>14\end{cases}
$$


Where $a_{n=1 \ldots 4}, b_{n=1 \ldots 4}, c_{n=1 \ldots 4}, d_{n=1 \ldots 4}, \quad=\quad$ polynomial $R=$ range from the scanner to the target

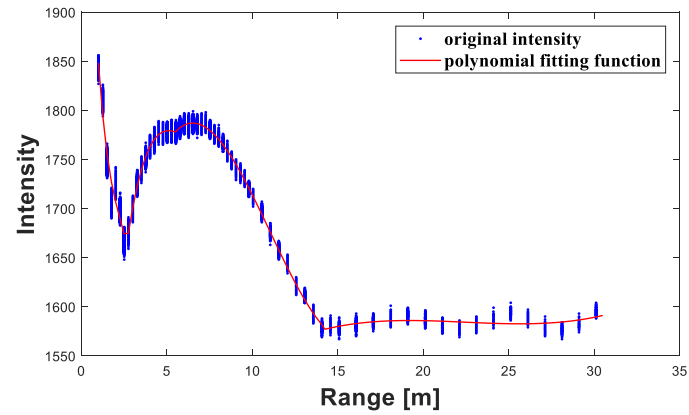

Figure 3. Original intensity with respect to range and fitted intensity-range function

Through polynomial fitting, the coefficients can be derived. The calculation values of each coefficient are listed in Table 2 .

\begin{tabular}{|c|c|c|c|c|}
\hline$n$ & $a$ & $b$ & $c$ & $d$ \\
\hline 1 & -36.1 & 249.2 & -635.8 & 2271 \\
2 & 4.06 & -71.5 & 412.5 & 996.7 \\
3 & 0.59 & -19.71 & 181 & 1280 \\
4 & 0.02 & -1.675 & 36.78 & 1321 \\
\hline
\end{tabular}

Table 2. Coefficients for fitted intensity-range function

The correction method is applied to the original intensity data. As shown in Figure 4, after range correction, the variation of intensity data from different ranges is greatly reduced.

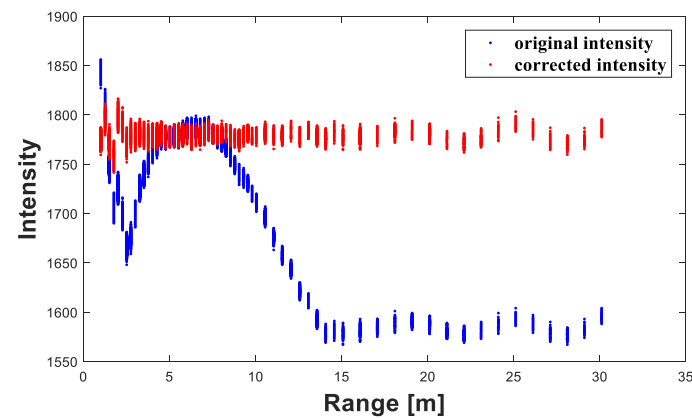

Figure 4. Original intensity data and intensity data after range effect correction

4.1.2 Incidence Angle Effect Correction: In order to derive the relationship between incidence angle and intensity, the incidence angel effect experiment was implemented at a fixed distance of 5 meters from the scanner. The target was set to rotate in steps of $5^{\circ}$ from $0^{\circ}$ to $80^{\circ}$. The scan resolution, the scan quality and field of view are set to be the same with the range effect experiment. The intensity data of the target are also extracted by Faro Scene manually. The result of the incidence angel effect experiment is illustrated in Figure 5. The intensity data decreases as the incidence angle increases so the estimation function can be written as follows:

$$
f_{2}(\theta)=a(\cos \theta)^{3}+b(\cos \theta)^{2}+c \cos \theta+d
$$

Where $\quad a, b, c, d=$ polynomial coefficients $\theta=$ incidence angle
Through polynomial fitting, the coefficients are derived and the calculation results are $a=345.3, b=-944.4, c=1173$ and $d=1193$.

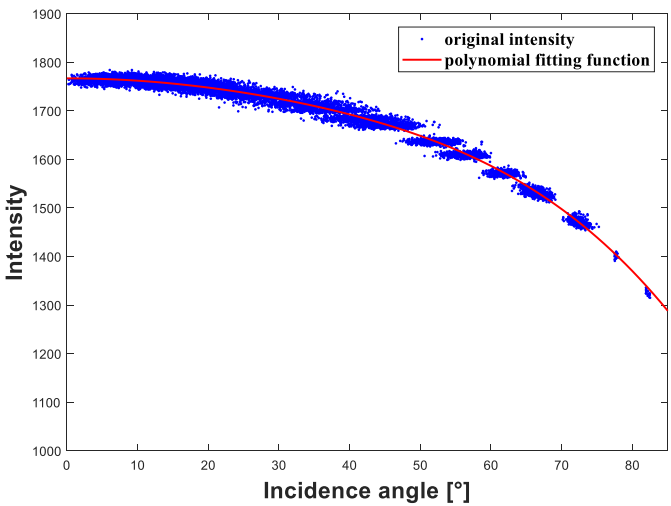

Figure 5. Original intensity with respect to incidence angle and fitted intensity-incidence angle function

The intensity data before and after correction are shown in Figure 6. After correction, the intensity data acquired at different incidence angles are approximately equal.

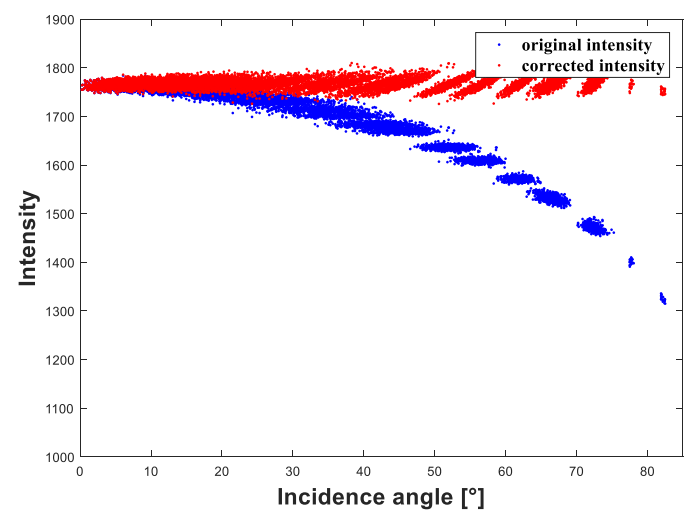

Figure 6. Original intensity data and intensity data after incidence angle effect correction

\subsection{Intensity Data Correction of the Study Areas}

The study areas chosen are some inner walls of an ancient Chinese pagoda, which has more than 1000 years' history. During its long history, its inner walls have certain deterioration due to both natural factors and human vandalism. As shown in Figure 7, these inner walls show different degrees of superficial detachment. The initial scanning surveys were for the 3D modelling of this pagoda.

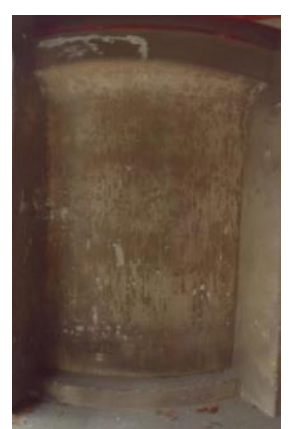

(a)

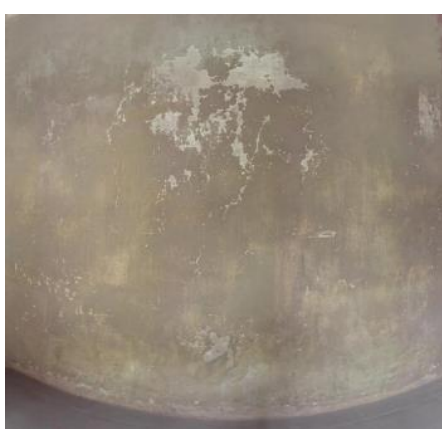

(b)
Figure 7. RGB images of some inner walls of the pagoda: (a) Wall 1; (b) wall 2 
The raw scanning data obtained by TLS was first pre-processed and those points that were not interested in this study were eliminated. Then the intensity data of the study areas were corrected through the correction models generated to diminish the range and incidence angle effects. As the visual appearance of the 3D point cloud intensity shown in Figure 8, the intensity variances of the homogenous region on wall 1 decrease greatly and the variances of the non-homogenous region increase after intensity correction. It could also be shown in Figure 9 that in general the intensity variances decrease with correction.

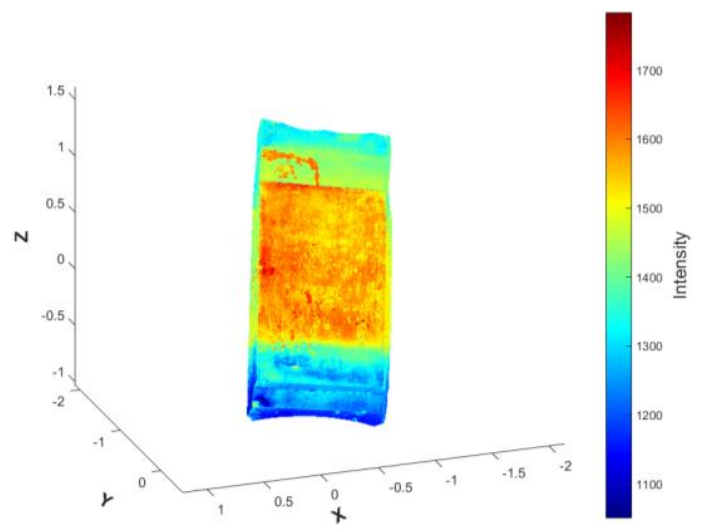

(a)

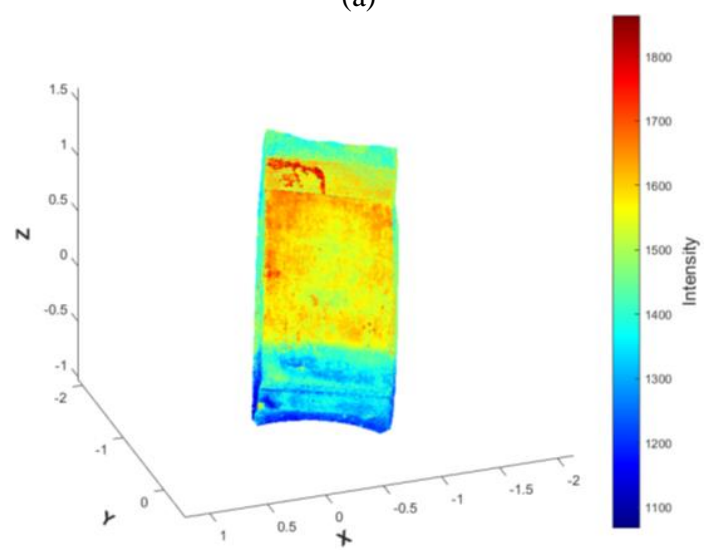

(b)

Figure 8. 3D point cloud intensity correction visualization: (a)

Wall 1 before correction; (b) wall 1 after correction;

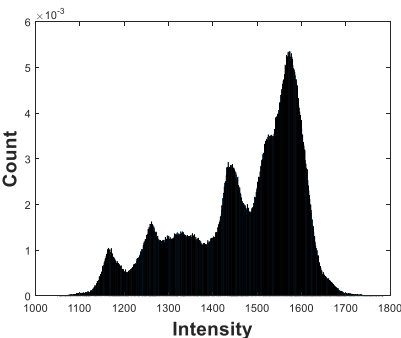

(a)

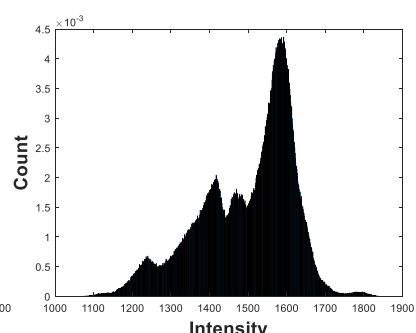

(b)
Figure 9. Histogram of intensity distribution of wall 1: (a) Original intensity; (b) corrected intensity;

\subsection{Damage Detection}

4.3.1 2D Intensity Image Generation: After intensity correction, the $3 \mathrm{D}$ point cloud was projected to generate the intensity image for classification based on the central projection method described in Section 3. The 3D point clouds with both the original intensity and corrected intensity are processed to generate 2D images. Figure 10 illustrates one of the intensity images generated.

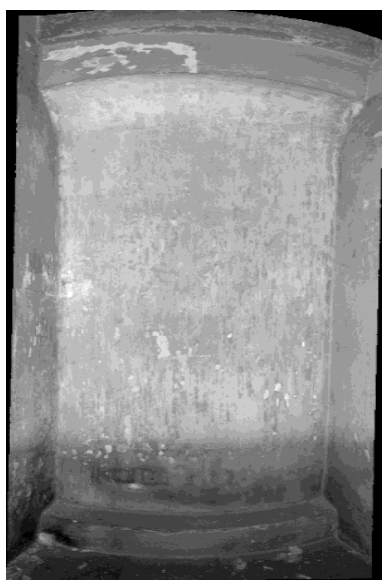

Figure 10. 2D intensity image generated of wall 1

4.3.2 Image Classification: K-means classification algorithm is utilised to classify the intensity images. For the classification algorithm, eight predefined classes were used. Figure 11 shows the classification results of wall 1. Figure 11(a) is the classification result of the original intensity and Figure 11(b) shows the damage areas detected. Figure 11(c) and 11(d) are the results of the corrected intensity. From visual assessment, both the original intensity and the corrected intensity can detect the damage areas. However, lots of wrongly-detected areas are found and some damage areas are undetected in the original intensity image. This is mainly because the original intensity is affected by several factors which makes it less accurate. Compared with the results of the original intensity, the classification results of the corrected intensity are more accurate. The major damage areas have been detected successfully and less wrongly-detected areas are found. However, there still exist some small damage areas that are failed to be detected. In Figure 12, the classification results of wall 2 are shown. Similar to the results of wall 1 , the classification result of the corrected intensity is more accurate than that of the original intensity. The experiment results indicate the validity of corrected intensity data in damage detection.

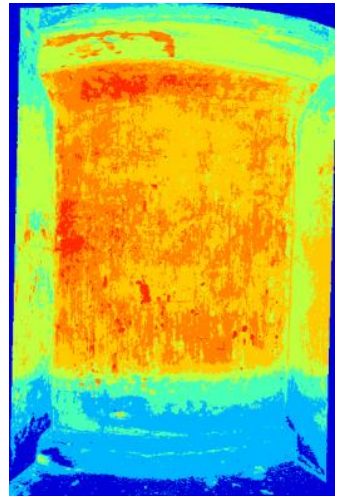

(a)

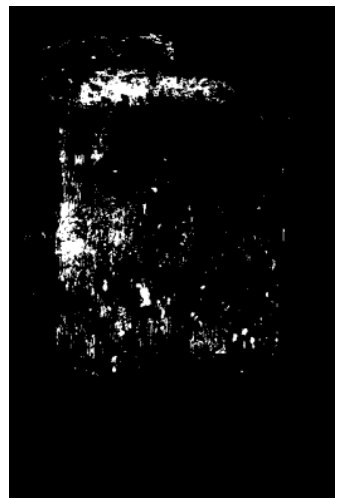

(b) 


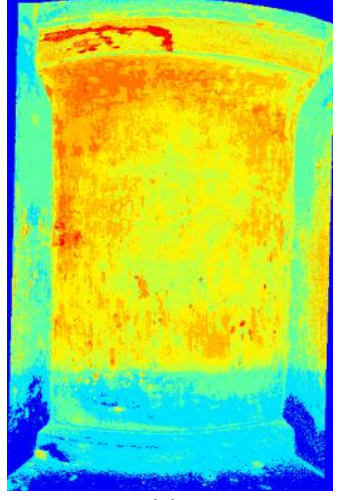

(c)

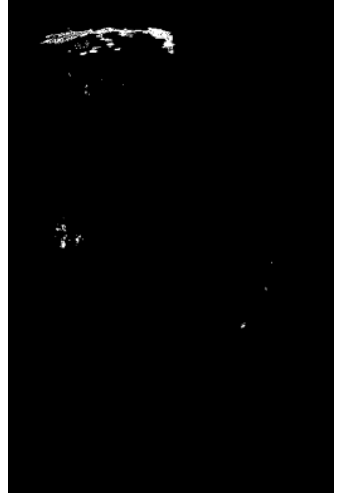

(d)
Figure 11. Classification results of wall 1 (8 clusters): (a) Original intensity; (b) damage areas detected using original intensity; (c) corrected intensity; (d) damage areas detected using corrected intensity

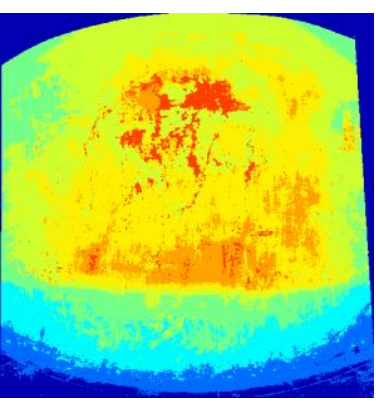

(a)

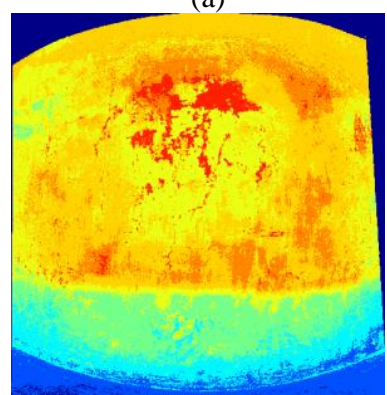

(c)

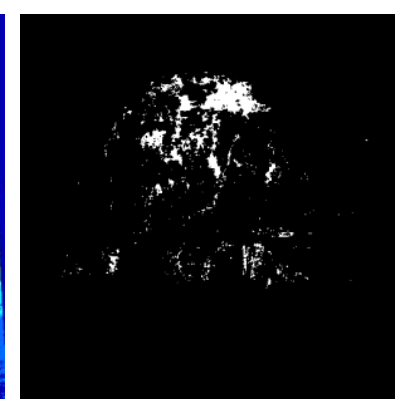

(b)

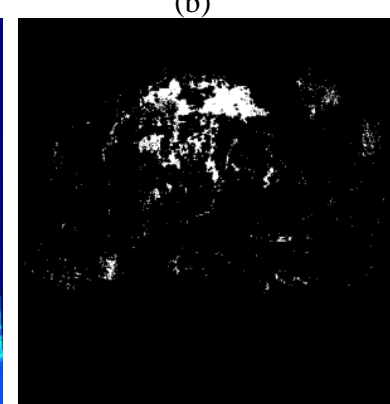

(d)
Figure 12. Classification results of wall 2 (8 clusters): (a) Original intensity; (b) damage areas detected using original intensity; (c) corrected intensity; (d) damage areas detected using corrected intensity

4.3.3 3D Point Cloud Re-projection: In order to re-project the detected damage areas to the $3 \mathrm{D}$ point cloud, the images of the damage areas detected are utilized. The pixel locations of every damage area are first acquired. In comparison to the data stored in the $2 \mathrm{D}$ projection process, the $3 \mathrm{D}$ coordinates of the damage areas can be derived. In Figure 13, the damage areas of both wall 1 and wall 2 are shown in the 3D point cloud.

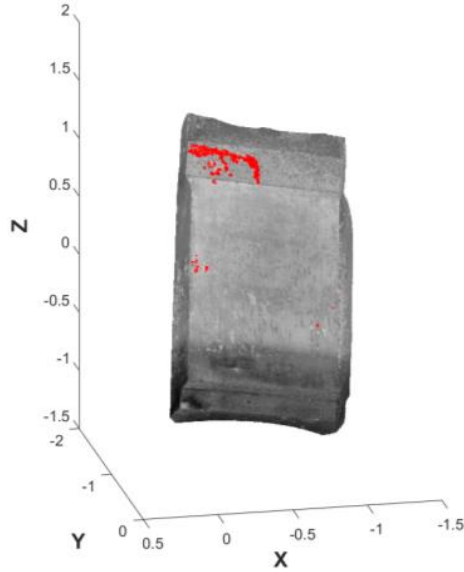

(a)

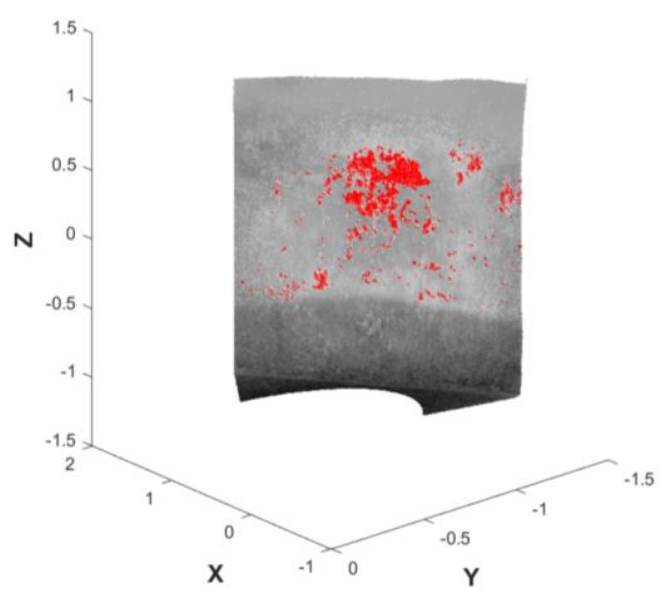

(b)

Figure 13. Damage areas visualization in 3D point clouds: (a) Wall 1; (b) wall 2

\section{CONCLUSIONS}

In this paper we presented a damage detection method for historical architecture based on corrected TLS intensity data. The proposed method takes the advantage of the 3D laser scanning data for digital documentation of historical sites which is more efficient and accurate to a certain extent. Moreover, it can directly provide the information of certain damage of the structure without aids of other instruments. The damage information detected may be helpful for the professionals of the conservation sector to have more comprehensive knowledge of the current state of the historical site and its level of decay. Besides, it can further be integrated in the 3D models of the historical sites for long-term assessment and monitoring. However, the proposed method still has certain limitations. Some small damage areas are undetected which may be due to the correction model used. The model in this paper is derived from scanning an A3 paper which is considered as a Lambertian target. In fact, the surface of the walls is not perfect Lambertian target. Therefore, a more accurate correction model which considers the target surface properties needs to be investigated. In addition, the damage detection process needs to go through 2D projection and $3 \mathrm{D}$ re-projection, which may be time consuming. Hence, in the future, the quantitative relationships between the intensity data and certain pathology need to be studied thus no further image processing is needed which can improve both the efficiency and accuracy of damage detection. 


\section{ACKNOWLEDGEMENTS}

The research leading to these results has been funded by Beijing Key Laboratory of Urban Spatial Information Engineering, NO. 2017216 and National Natural Science Foundation of China (NSFC) (41671449)

\section{REFERENCES}

Ahokas, E., Kaasalainen, S., Hyyppä, J., and Suomalainen, J. 2006. Calibration of the Optech ALTM 3100 laser scanner intensity data using brightness targets. International Archives of Photogrammetry, Remote Sensing and Spatial Information Sciences, 36(Part 1), pp. 1Á6.

Armesto-González, J., Riveiro-Rodríguez, B., GonzálezAguilera, D., and Rivas-Brea, M. T. 2010. Terrestrial laser scanning intensity data applied to damage detection for historical buildings. Journal of Archaeological Science, 37(12), 3037-3047.

Blaskow, R., and Schneider, D. 2014. Analysis and correction of the dependency between laser scanner intensity values and range. The International Archives of Photogrammetry, Remote Sensing and Spatial Information Sciences, 40(5), 107.

Carrea, D., Abellan, A., Humair, F., Matasci, B., Derron, M. H., and Jaboyedoff, M. 2016. Correction of terrestrial lidar intensity channel using oren-nayar reflectance model: an application to lithological differentiation. Isprs Journal of Photogrammetry \& Remote Sensing, 113, 17-29.

Coren, F., and Sterzai, P. 2006. Radiometric correction in laser scanning. International Journal of Remote Sensing, 27(15), pp. 3097-3104.

Ding, Q., Chen, W., King, B., Liu, Y., and Liu, G. 2013. Combination of overlap-driven adjustment and phong model for lidar intensity correction. Isprs Journal of Photogrammetry \& Remote Sensing, 75(1), 40-47.

Fang, W., Huang, X., Zhang, F., and Li, D. 2014. Intensity correction of terrestrial laser scanning data by estimating laser transmission function. IEEE Transactions on Geoscience \& Remote Sensing, 53(2), 942-951.

Garcíatalegón, J., Calabrés, S., Fernándezlozano, J., Iñigo, A. C., Herrerofernández, H., and Ariaspérez, B., et al. 2015. Assessing pathologies on villamayor stone (salamanca, spain) by terrestrial laser scanner intensity data. ISPRS - International Archives of the Photogrammetry, Remote Sensing and Spatial Information Sciences, XL-5/W4, 445-451.

Habib, A. F., Kersting, A. P., Shaker, A., and Yan, W. Y. 2011. Geometric calibration and radiometric correction of lidar data and their impact on the quality of derived products. Sensors, 11(9), 9069.

Hartzell, P., Glennie, C., Biber, K., and Khan, S. 2014. Application of multispectral lidar to automated virtual outcrop geology. Isprs Journal of Photogrammetry \& Remote Sensing, 88(1), 147-155.

Höfle, B., and Pfeifer, N. 2007. Correction of laser scanning intensity data: data and model-driven approaches. Isprs Journal of Photogrammetry \& Remote Sensing, 62(6), 415-433.
Jutzi, B., and Gross, H. (2014). Normalization of lidar intensity data based on range and surface incidence angle. Comptes Rendus Mecanique, 6(6), 407-414.

Kaasalainen, S., Jaakkola, A., Kaasalainen, M., Krooks, A., and Kukko, A. 2011. Analysis of incidence angle and distance effects on terrestrial laser scanner intensity: Search for correction methods. Remote Sensing, 3(10), pp. 2207-2221.

Kashani, A. G., Olsen, M. J., Parrish, C. E., and Wilson, N. 2015. A Review of LiDAR radiometric processing: From Ad Hoc intensity correction to rigorous radiometric calibration. Sensors, 15(11), pp. 28099-28128.

Kedzierski, M., Walczykowski, P., Wojtkowska, M., and Fryskowska, A. 2017. Integration of point clouds and images acquired from a low-cost nir camera sensor for cultural heritage purposes. ISPRS - International Archives of the Photogrammetry, Remote Sensing and Spatial Information Sciences, XLII-2/W5, 407-414.

Lerones, P. M., Vélez, D. O., Rojo, F. G., Gómezgarcíabermejo, J., and Casanova, E. Z. 2016. Moisture detection in heritage buildings by $3 \mathrm{~d}$ laser scanning. Studies in Conservation (supp 1), 160120002416003 .

Li, Z., Jupp, D. L., Strahler, A. H., Schaaf, C. B., Howe, G., Hewawasam, K., ... and Saenz, E. J. 2016. Radiometric Calibration of a Dual-Wavelength, Full-Waveform Terrestrial Lidar. Sensors, 16(3), pp. 313.

Moses, C., Robinson, D., and Barlow, J. 2014. Methods for measuring rock surface weathering and erosion: a critical review. Earth-Science Reviews, 135(4), 141-161.

Nield, J. M., King, J., and Jacobs, B. 2014. Detecting surface moisture in aeolian environments using terrestrial laser scanning. Aeolian Research, 12(12), 9-17.

Riveiro, B., Lourenço, P. B., Oliveira, D. V., González-Jorge, H., and Arias, P. 2016. Automatic morphologic analysis of quasi-periodic masonry walls from lidar. Computer-Aided Civil and Infrastructure Engineering, 31(4), 305-319.

Tan, K., and Cheng, X. 2016. Correction of incidence angle and distance effects on tls intensity data based on reference targets. Remote Sensing, 8(3), 251.

Tan, K., Cheng, X., Ding, X., and Zhang, Q. 2016. Intensity data correction for the distance effect in terrestrial laser scanners. IEEE Journal of Selected Topics in Applied Earth Observations \& Remote Sensing, 9(1), 304-312.

Zhu, X., Wang, T., Darvishzadeh, R., Skidmore, A. K., and Niemann, K. O. 2015. 3d leaf water content mapping using terrestrial laser scanner backscatter intensity with radiometric correction. Isprs Journal of Photogrammetry \& Remote Sensing, 110, 14-23. 